\title{
Automated Production of Human Induced Pluripotent Stem Cell-Derived Cortical and Dopaminergic Neurons with Integrated Live-Cell Monitoring
}

\begin{abstract}
Ashutosh Dhingra ${ }^{*}$, , Joachim Täger $^{*}{ }^{1}$, Elisangela Bressan ${ }^{*}{ }^{1}$, Salvador Rodriguez-Nieto ${ }^{1}$, Manmeet-Sakshi Bedi ${ }^{1}$, Stefanie Bröer $^{1}$, Eldem Sadikoglou ${ }^{1}$, Noémia Fernandes ${ }^{2}$, Melissa Castillo-Lizardo ${ }^{1}$, Patrizia Rizzu ${ }^{2}$, Peter Heutink ${ }^{1,3}$

${ }^{1}$ Genome Biology of Neurodegenerative Diseases, German Center for Neurodegenerative Diseases (DZNE) ${ }^{2}$ Applied Genomics for Neurodegenerative Diseases, German Center for Neurodegenerative Diseases (DZNE) ${ }^{3}$ Department for Neurodegenerative Diseases, Hertie Institute for Clinical Brain Research, University of Tübingen

*These authors contributed equally
\end{abstract}

\section{Corresponding Author}

Ashutosh Dhingra

ashutosh.dhingra@dzne.de

\section{Citation}

Dhingra, A., Täger, J., Bressan, E., Rodriguez-Nieto, S., Bedi, M.S.,

Bröer, S., Sadikoglou, E., Fernandes, N., Castillo-Lizardo, M., Rizzu, P.,

Heutink, P. Automated Production of Human Induced Pluripotent Stem CellDerived Cortical and Dopaminergic Neurons with Integrated Live-Cell Monitoring. J. Vis. Exp. (162), e61525, doi:10.3791/61525 (2020).

\section{Date Published}

August 6, 2020

DOI

$10.3791 / 61525$

URL

jove.com/video/61525

\section{Abstract}

Manual culture and differentiation protocols for human induced pluripotent stem cells (hiPSC) are difficult to standardize, show high variability and are prone to spontaneous differentiation into unwanted cell types. The methods are labor-intensive and are not easily amenable to large-scale experiments. To overcome these limitations, we developed an automated cell culture system coupled to a high-throughput imaging system and implemented protocols for maintaining multiple hiPSC lines in parallel and neuronal differentiation. We describe the automation of a short-term differentiation protocol using Neurogenin-2 (NGN2) over-expression to produce hiPSC-derived cortical neurons within 6-8 days, and the implementation of a long-term differentiation protocol to generate hiPSC-derived midbrain dopaminergic (mDA) neurons within 65 days. Also, we applied the NGN2 approach to a small molecule-derived neural precursor cells (smNPC) transduced with GFP lentivirus and established a live-cell automated neurite outgrowth assay. We present an automated system with protocols suitable for routine hiPSC culture and differentiation into cortical and dopaminergic neurons. Our platform is suitable for long term hands-free culture and high-content/ high-throughput hiPSC-based compound, RNAi and CRISPR/Cas9 screenings to identify novel disease mechanisms and drug targets.

\section{Introduction}

Human induced pluripotent stem cells (hiPSC) are selfrenewing and can differentiate in almost any adult cell type. These characteristics make hiPSC a useful tool for disease modeling in basic research and drug discovery ${ }^{1}$. Human iPSC retains the donor genetic background which allows deriving disease-relevant cell types that are most 
affected/involved in the disease course, for example, different neuronal subtypes for neurodegenerative diseases ${ }^{2}, 3$. Also, hiPSC overcomes some of the limitations of animal and cellular over-expression models by modeling diseases in a human context and physiological protein expression levels, and have proven to be a valuable asset in modeling diseases ranging from monogenic, complex and epigenetic disorders as well as late-onset diseases ${ }^{4}$.

Despite these benefits and opportunities, several limitations of hiPSC still need to be addressed. Current hiPSC culture and differentiation protocols are not cost-effective, difficult to standardize and are labor-intensive. Manual culture steps can result in high variability in the yields and phenotypes due to differences in growth and spontaneous differentiation of hiPSC. Therefore, experimenter-dependent variation needs to be reduced by implementing more standardized handling techniques and simplifying protocols which can be achieved using automation ${ }^{5}$. The establishment of automated hiPSC culture and differentiation protocols will set common standards for both academic and industrial research projects, and allow the generation of biologically relevant disease models and more reproducible results.

Previous work has attempted automation of hiPSC cultures $^{6,7,8}$ but their protocols have been restricted to specific cell culture plate formats dependent on the system and lacking adaptability to different assay formats. Such systems are useful in the bulking of cells but may not be suitable for automated differentiation into desired cell types, disease phenotyping, and screening purposes. Additionally, a large-scale automated platform for fibroblast derivation, hiPSC generation and differentiation has been described ${ }^{9}$ but on a scale that can only be achieved by high-throughput laboratories dedicated to the production of lines which seems attractive but can be unaffordable for many academic laboratories.

We developed a fully automated cell culture system based on a liquid handling station in a High-Efficiency Particulate Air (HEPA)-filtered environment in conjunction with a largecapacity $\mathrm{CO}_{2}$ incubator, a brightfield imaging cytometer and a robotic arm for plate transport. These components provide the basis for stable and reproducible hiPSC culture and differentiation. We complemented the system with an automated $-20{ }^{\circ} \mathrm{C}$ storage system for compound or virus storage and a high-speed spinning disk confocal live-cell imager. Custom-made protocols were generated allowing automated cell seeding, media changes, confluency checks, cell expansion and assay plate generation with sample treatment and plate imaging, making the system compatible with high-content/high-throughput screenings. The automated cell culture and imaging system are operated using the controlling software and the custom-made graphical user interface (GUI). The GUI allows users to import CSV files containing cell line-specific parameters needed for method execution. Additionally, the GUI enables to schedule numerous experiments in any sequence using the built-in calendar view thus allowing full control of the time when each method starts.

Our automated cell culture system uses standardized pipetting speeds, passaging times, confluency thresholds, seeding densities, and medium volumes with the flexibility to culture cells in a variety of plate formats (96-, 48-, 24-, 12-, 6- or 1-well plate format). We adapted a recently published short-term differentiation protocol for converting hiPSC into neurons that can yield TUBB3 positive neurons in 6 days $^{10,11}$. We also established the automated differentiation and imaging of small molecule neural precursor cells 
(smNPC) into neurons constitutively expressing GFP under EF1a promoter $^{12}$ and iPSC into midbrain dopaminergic (mDA) neurons, adapting a previously published dual-SMAD inhibition protocol ${ }^{13}$ that yields mDA neurons within 65 days.

\section{Protocol}

\section{Basic procedures for automating cell cultures and imaging}

\section{Load new culture plates and tips}

1. Open the GUI and click on the Resource/Instrument process view button. To run any resource/instrument process, select the resource/instrument process and click on the Run Instrument process button.

2. Run the resource process RunHepaHood and Reloading (see step 1.1.1). Open the door, load the cell culture plates and disposable tips in the corresponding position as indicated in the pop-up image on the controlling PC.

3. Wipe the door with $70 \%$ ethanol for decontamination and close it. Run the instrument process Decontamination from the GUI (see step 1.1.1). The system gets sterilized by UV radiation for $30 \mathrm{~min}$.

\section{Refill culture media and/or dissociation reagent}

1. Execute the instrument step RunHepahood (see step 1.1.1). Open the front door of the liquid handling station. Decontaminate reservoirs (containing media, PBS and/or dissociation reagent) with $70 \%$ ethanol and place them on the deck to the assigned positon (as defined in cfg.csv file). De-lid the reservoirs.

2. Decontaminate the door with $70 \%$ ethanol and close it. Power down the HEPA hood by running the resource/instrument process "InitHepaHood" from the GUI.

\section{Create a new "Cell line" and project in the GUI}

1. Create/modify the user-defined "Cell line"-files consisting of the Config (".cfg.csv), the import $\left({ }^{*}\right.$.imp.csv), the resource ( ${ }^{*}$.rsv.csv) and the workflow ( ${ }^{*}$.wfl.csv) files.

2. Open the GUI and create a new "Cell line" by clicking on the Add cell line button in the "Cell line editor" view. A wizard opens where the Config file needs to be selected and a project name with a short description needs to be entered.

3. Navigate through this wizard using the green arrowhead and confirm the settings by clicking on the green checkmark. Create a new project for the generated "Cell line" by clicking on the Add project button.

4. Enter a project name and description and define a project color that will be visible in the calendar view of the GUI. Navigate to the next page of the wizard by clicking on the arrowhead.

5. Create a new batch by clicking on the Add batch button and giving a short name and description in the pop-up window. Select all process steps and schedule the start time for each of the process step. Close the window by clicking on OK.

\section{Execute an automated method using the GUI}

1. Go to the calendar view of the GUI and click on the Add process step button.

2. Select the Cell line and after navigating to the next page of the wizard choose the project. Mark the batch to be used and click on the arrowhead pointing to the 
right. Depending on the method used, batches have to be either empty (for receiving new plates), or contain culture plates or assay plates (all other processes).

3. Navigate to the next page of the wizard and select the process step which shall be executed. Go to the last page of this wizard and schedule the experiment. In the section "Parameter Details" variables that are needed to run the method can be modified. Confirm by clicking OK.

5. Loading of culture and assay plates into the $\mathrm{CO}_{2}$ incubator

NOTE: 1-well plates are defined as culture plates since they are commonly used to bulk cells. All multi-well plates are defined as assay plates.

1. Execute the instrument process "RunHepaHood" from the GUI as described in step 1.1.1. and open the door in front of the robotic arm.

2. Wipe the bottom of the assay plates with $70 \%$ ethanol and a lint-free tissue if plates are loaded for automated imaging. Place culture or assay plates on the left shelf. Make sure that the orientation of the plate is correct. For assay plates, well $\mathrm{A} 1$ has to point towards the robotic arm while the edges of culture plates have to point to the right.

3. Wipe the door with $70 \%$ ethanol for decontamination and close it.

4. Execute the method "Loading Of Culture Plates" for importing 1-well plates or "Loading Of Assay Plates" for importing multi-well plates as described in step 1.4. Use an empty batch to run both methods. If plate barcodes are already present in this batch, deselect them by clicking on the checkboxes.
5. Transport individual plates from the shelf to the $\mathrm{CO}_{2}$ incubator platform using the robotic arm. The builtin plate shuttle station retrieves the plate and stores them in one of the racks of the $\mathrm{CO}_{2}$ incubator. The cells are maintained at $37^{\circ} \mathrm{C}$ and $5 \% \mathrm{CO}_{2}$.

\section{Unloading of plates from the $\mathrm{CO}_{2}$ incubator}

1. Execute the method "Unload plates" (see step 1.4). Select the batch containing the plates which need to be exported. Individual plates can be selected by their barcodes.

2. Transport the plates out from the $\mathrm{CO}_{2}$ incubator to the left shelf using the robotic arm.

3. Start the HEPA hood by using the instrument process "RunHepaHood" as described in step 1.1.1. and open the door in front of the robotic arm. Remove the plates, decontaminate the door with $70 \%$ ethanol before closing it and power down the HEPA hood.

\section{Automation protocols}

\section{Seeding of plates from tubes}

1. Start the HEPA hood by executing the instrument process RunHepaHood (see step 1.1.1). Open the door in front of the liquid handling station. Input the $50 \mathrm{~mL}$ tube containing the cell suspension and de-lid the tube.

2. Open the door in front of the robotic arm and load coated culture plates for receiving cells on the shelf. Decontaminate and close both doors. Execute the method "Seeding of plates from tubes" (see step 1.4).

3. Count cells at the brightfield imaging cytometer using direct cell counting function. For cell counting, use 16 wells as replicates in a 384-well plate format. 
4. Transport the 384-well counting plate from the deck to the brightfield imaging cytometer via the turntable using the robotic arm and start the imaging process. The cytometer automatically determines the cell number per milliliter. Bring the counting plate back to its original position using the robotic arm.

5. Transport a coated culture or assay plate from the shelf to the pipetting deck using the robotic arm. Remove coating and seed cells in the user-defined number, and volume suitable for the plate format (see Table 1). Move the plate to the on-deck shaker for 10 $\mathrm{s}$ at $500 \mathrm{rpm}$ for cell distribution and transfer to the $\mathrm{CO}_{2}$ incubator.

\section{Automated confluence assessment}

1. Execute the method "Check Confluency" as described in step 1.4. Select a batch that contains at least one culture plate and no assay plates. In the section "Parameter Details" input "iPSCf_2020" for image acquisition and imaging analysis settings.

2. Transport the first plate from the $\mathrm{CO}_{2}$ incubator to the brightfield imaging cytometer via the turntable using the robotic arm.

3. Perform imaging of cells in the brightfield imaging cytometer for confluence check of hiPSC colonies. Use "confluence" application and image 13 fields of the 1-well pate and automatically calculate the average area occupied by cells.

4. Transport the plate back to the $\mathrm{CO}_{2}$ incubator using the robotic arm. Repeat steps 2.2.2. to 2.2.4. for the remaining plates.

\section{Media change of culture plates or assay plates}

1. Execute the method "Media Change Of Culture Plates" as described in step 1.4. and select a batch containing only culture plates. Set the variable indicating whether tips or needles are used for the pipetting steps in the section "Parameter Details". For media change of any multi-well plate, execute the method "Media Change Of Assay Plates" and select a batch containing assay plates.

2. Transport the individual plates from the $\mathrm{CO}_{2}$ incubator to the deck using the robotic arm and delid the plates. Automatically tilt the plates and aspirate old media and discard it into the waste collection module. Add $12 \mathrm{~mL}$ of fresh media. Re-lid the plate and transport the plates back to the $\mathrm{CO}_{2}$ incubator using the robotic arm.

\section{Subcultivation}

NOTE: Execute the instrument process "RunHepaHood" from the GUI as described in 1.1.1. and open the front door of the liquid handling station. Load media and dissociation reagent at the required positions (see step 1.2). If tips need to be refilled, use the "Reloading" as described in step 1.1. Input a $50 \mathrm{~mL}$ tube for receiving the cell suspension and de-lid the tube.

1. Execute the method "Subcultivation Of Adherent Cells" (see step 1.4). Select the batch containing culture plates that need subcultivation.

2. Transport the plates from the $\mathrm{CO}_{2}$ incubator to the deck using the robotic arm and de-lid the plates. Automatically tilt the plates and remove old media and discard it into the waste collection module. Wash cells once with $8 \mathrm{~mL}$ of PBS. Add $8 \mathrm{~mL}$ of $0.5 \mathrm{mM}$ EDTA for clump based passaging. Incubate on the deck for $8 \mathrm{~min}$. 
3. Remove EDTA solution from the plates and discard it into the waste collection module. Add $12 \mathrm{~mL}$ of fresh medium and transport the plates to the shaker. Shake at $2000 \mathrm{rpm}$ for $1 \mathrm{~min}$ to dislodge the colonies.

4. Triturate the cell suspension for five cycles of pipetting to break the iPSC colonies into a smaller size ( $50-80$ $\mu \mathrm{m})$. Transfer the cell suspension into a $50 \mathrm{~mL}$ tube on the deck. Seed cells automatically as described in step 2.1.5 using a split ratio of 1 in 7 .

NOTE: Optional, single cell passaging. Generate a single cell suspension using single cell dissociation reagent (see Table of Materials). Instead of 0.5 mM EDTA in step 2.4.2., use $8 \mathrm{~mL}$ of single cell dissociation reagent and incubate for $20 \mathrm{~min}$ at 37 ${ }^{\circ} \mathrm{C}$. Collect the cell suspension into a $50 \mathrm{~mL}$ tube and add equal volume of media. Dissociation using the single cell dissociation reagent requires a manual step to pellet the cells. Centrifuge cells at $300 \times g$ for $3 \mathrm{~min}$. Remove the supernatant and resuspend the cell pellet in $12 \mathrm{~mL}$ of the required media and proceed with "Seeding of plates from tubes" (see step 2.1). Supplement the media with $2 \mu \mathrm{M}$ thiazovivin on the day of seeding when using single cell suspension of hiPSCs.

\section{Automated high-content, high throughput imaging}

NOTE: The measurement setting has to be available (see Supplementary File 1: step 1.1). The assay plates to be imaged are available in the incubator.

1. Execute the method "Imaging" (see step 1.4). Select a batch that contains at least one assay plate and no culture plate. In the section "Parameter Details", enter the plate type, the plate definitions (for standard 96-well imaging plates: "Plate-96-20170918113842") and the name of the measurement setting.

2. Transport the assay plate via the turntable to the automated confocal microscope using the robotic arm to start the imaging process. Recover the plate at the end of the imaging from the microscope and transport it back to the $\mathrm{CO}_{2}$ incubator. Repeat the process of the remaining plates in the batch.

\section{Automated maintenance and expansion of hiPSC}

1. The sequence of automated confluency checks, media changes and subcultivation

1. Seed cells on 1-well plates using "Seeding of plates from tubes" method (see step 2.1). Alternatively, import manually seeded 1-well plates using "Loading of culture plate" method (see step 1.5).

2. Schedule automated confluency checks daily to monitor colony growth from day 1 to day 6 of the culture for iPSC (see step 2.2).

3. Refresh media every second day using the "Media change of culture plates" method (see step 2.3). 12 $\mathrm{mL}$ of medium is used per plate.

4. On day 6, start the "Subcultivation" process (see step 2.4.).

\section{Automated differentiation}

1. Human iPSC to cortical NGN2 neurons

NOTE: Manual hiPSC preparation. The protocol involves over-expression of NGN2 using lentiviral vectors for delivery. Transduce hiPSC with NGN2 to rTTA3 lentivirus in a 1:2 ratio $\left(>\sim 10^{7} \mathrm{TU} / \mathrm{mL}\right)$. Cells are then selected for one passage with $0.5 \mu \mathrm{g} / \mathrm{mL}$ puromycin. A detailed 
protocol for generating stable hiPSC-NGN2 lines is in the

Supplementary File 1: step 2.

1. Proceed with the "Subcultivation" process as described using the single cell dissociation reagent described in note of step 2.4.4. when hiPSC reach 70$80 \%$ confluency

2. Remove the supernatant and resuspend the cell pellet in $12 \mathrm{~mL}$ of NGN2 media (Table of Materials) containing $2.5 \mu \mathrm{g} / \mathrm{mL}$ doxycycline (dox) and $2 \mu \mathrm{M}$ thiazovivin.

3. Start differentiation using the "Seeding of plates from tubes" method (see step 2.1). Set the desired seeding density of iPSC to $30,000 / \mathrm{cm}^{2}$ (see table 1). The iPSC are plated onto pre-coated plates with $0.1 \mathrm{mg} /$ $\mathrm{mL}$ poly-L-ornithine (PLO) and $5 \mu \mathrm{g} / \mathrm{mL}$ laminin.

NOTE: 1-well plates are preferred for RNA and proteomics-based studies while the 96-well plate format is preferred for imaging experiments. Other assay plate formats such as 48-, 24-, 12- or 6-well plates may also be used.

4. On day 1 of differentiation, Refresh media using the "Media change of assay plates (see step 2.3) using NGN2 medium, supplemented with $2.5 \mu \mathrm{g} /$ $\mathrm{mL}$ dox and $10 \mu \mathrm{M} \mathrm{N}$-[2S-(3,5-difluorophenyl)acetyl]L-alanyl-2-phenyl-glycine, 1,1-dimethylethyl ester (DAPT). Continue with media changes every 2-3 days until the desired day of differentiation (see step 2.3).

5. On day 4 of differentiation, carry out another media change (see step 2.3.) using NGN2 medium supplemented with $10 \mathrm{ng} / \mathrm{mL}$ brain-derived neurotrophic factor (BDNF), $10 \mathrm{ng} / \mathrm{mL}$ glial cellderived neurotrophic factor (GDNF) and $10 \mathrm{ng} /$
$\mathrm{mL}$ neurotrophic factor 3 (NT-3) to enhance the maturation. At the end of the experiment, export the culture plates from the system for downstream experiments (see step 1.6).

2. Small molecule neural precursor cells (smNPC) to NGN2 neurons

NOTE: Derive smNPC following the adapted version of published protocol $^{12}$ (see Supplementary File 1: step 5). Modify the smNPC with NGN2 and rTTA3 virus (see Supplementary File 1: step 2). One round of NGN2 and rTTA transduction is sufficient to generate a stable population. Further modify smNPC with pLVXEF1a-AcGFP1-N1 lentivirus allowing to perform live-cell monitoring. A multiplicity of infection (MOI) of 10 is used for GFP lentiviral transduction.

1. Passage smNPC on reaching confluency using single cell dissociation reagent as described in step 2.4. Note.

2. Seed cells using the automated cell culture system at a cell density of $50,000 / \mathrm{cm}^{2}$ using "Seeding of plates from tubes" method (see step 2.1.) onto pre-coated plates with $0.1 \mathrm{mg} / \mathrm{mL}$ PLO, and $5 \mu \mathrm{g} / \mathrm{mL}$ laminin in NGN2 medium supplemented with $2.5 \mu \mathrm{g} / \mathrm{mL}$ dox.

3. On day 3 , perform a media change (see step 1.3.) using fresh NGN2 medium containing $2.5 \mu \mathrm{g} / \mathrm{mL}$ dox and $10 \mu \mathrm{M}$ DAPT. After day 3, perform media changes every third day with fresh NGN2 medium containing $2.5 \mu \mathrm{g} / \mathrm{mL}$ dox, BDNF, GDNF and NT-3 at $10 \mathrm{ng} / \mathrm{mL}$ each.

4. Image cells daily using the "Automated high content, high throughput imaging" method (see step 2.5) for monitoring differentiation status using GFP as readout for neurite outgrowth. Set the laser power to $80 \%$ 
and use an exposure time of $30 \mathrm{~ms}$. Acquire a large number of fields (e.g., 25 fields) using $20 x$ objective.

\section{Batch image analysis}

1. Perform image analysis with image analysis software 1 , using the neurite outgrowth template.

2. Open the software. Select the "Data" option and browse to the imaging data folder, click ok to load data to be analyzed. Click open and from the top menu bar, select protocol and from the application menu select neurite outgrowth. Go to "algorithms" and use the "488" channel to define the nucleus, cell body and neurite. Adjust the threshold parameters for each.

3. Select the wells to be used for image analysis. On the bottom right click link and select features to be analyzed such as cell count average, total skeleton length total. Proceed with "pre-analyze" followed by "preview".

4. Check if the preview settings are acceptable and start the analysis in the batch mode. The results are available in the parent folder under "reports" in .csv file format that can be opened in excel.

NOTE: Image analysis script is available upon request.

5. Plot average neurite length obtained by total neurite length normalized to cell number.

\section{Automated differentiation of hiPSC into midbrain dopaminergic (mDA) neurons}

\section{Manual hiPSC preparation}

1. Dissociate $70-80 \%$ confluent hiPSC into single cells using the single cell dissociation reagent. Briefly, incubate cells with single cell dissociation reagent $\left(100 \mu \mathrm{L} / \mathrm{cm}^{2}\right)$ for $30 \mathrm{~min}$ at $37{ }^{\circ} \mathrm{C}$, collect cell suspension into a conical tube, centrifuge at $200 \times g$ for $5 \mathrm{~min}$ and resuspend cell pellet in iPSC culture medium.

2. Seed 200,000 cells $/ \mathrm{cm}^{2}$ on extracellular matrixcoated 1-well plates and iPSC culture medium supplemented with $10 \mu \mathrm{M}$ Y-27632. Culture cells overnight at $37^{\circ} \mathrm{C}$ and $5 \% \mathrm{CO}_{2}$.

\section{Automated differentiation: Phase 1}

1. Prepare the automated culture system as described in step 1.1-1.2.

2. Load culture plates containing cells into the $\mathrm{CO}_{2}$ incubator of the automated culture system (see step 1.5).

3. Prepare KSR medium (see Table of Materials) and supplement with small molecules (see Table 2) required for starting day 0 of differentiation. Use only freshly prepared media with small molecules and growth factors.

4. Perform media changes of the culture plates as described in step 2.3 on days 0 and 1 of differentiation and then every second day until day 25 .

5. From day 5, shift media formulation gradually as described in detail in Table 3.

6. On day 11 , add $\mathrm{mDA}$ neuron differentiation medium supplemented with CHIR (until day 13), BDNF, AA1, GDNF, db-CAMP, TGFß3 and DAPT (see the Table of Materials).

7. On day 25 , unload plates (see step 1.6.)

\section{Manual replating 1}

1. Dissociate day $25 \mathrm{mDA}$ precursors into single cells using the single cell dissociation reagent. Briefly, 
incubate cells with single cell dissociation reagent $\left(100 \mu \mathrm{L} / \mathrm{cm}^{2}\right)$ for $40 \mathrm{~min}$ at $37{ }^{\circ} \mathrm{C}$, collect cell suspension into a conical tube, centrifuge at $200 \times \mathrm{g}$ for $5 \mathrm{~min}$ and resuspend cell pellet in $\mathrm{mDA}$ neuron differentiation medium.

2. Seed 400,000 cells $/ \mathrm{cm}^{2}$ in 1-well culture plates precoated with $0.1 \mathrm{mg} / \mathrm{mL}$ PLO, $10 \mu \mathrm{g} / \mathrm{mL}$ laminin and $2 \mu \mathrm{g} / \mathrm{mL}$ fibronectin in mDA neuron differentiation medium supplemented with $10 \mu \mathrm{M}$ Y-27632 (until day 26) and small molecules and growth factors described in Table 2. Culture cells overnight at $37{ }^{\circ} \mathrm{C}$ and $5 \%$ $\mathrm{CO}_{2}$.

\section{Automated differentiation: Phase 2}

1. Load culture plates containing mDA neurons into the $\mathrm{CO}_{2}$ incubator of the automated culture system as described in step 1.5

2. Prepare $\mathrm{mDA}$ neuron differentiation medium (see Table of Materials) and supplement with small molecules and growth factors required for the final differentiation from day 26 onwards (see Table 2).

3. Perform media changes of culture plates as described in step 2.3 on day 26 of differentiation and then every 3-4 days until day 65.

NOTE: For high-throughput imaging purposes, it is recommended to replate the mDA neurons in 96-well plates at low density on day 32 of differentiation, as described in step 5.5 .

\section{Manual replating 2}

1. Unload culture plates as described in step 1.6. Dissociate day $32 \mathrm{mDA}$ neurons into single cells as described in step 5.3.1.
2. Seed 100,000 cells $/ \mathrm{cm}^{2}$ in 96 -well plates pre-coated with $0.1 \mathrm{mg} / \mathrm{mL}$ PLO, $10 \mu \mathrm{g} / \mathrm{mL}$ laminin and $2 \mu \mathrm{g} /$ $\mathrm{mL}$ fibronectin in $\mathrm{mDA}$ neuron differentiation medium supplemented with $10 \mu \mathrm{M}$ Y-27632 (until day 33) and small molecules and growth factors (see Table 2). Culture cells overnight at $37^{\circ} \mathrm{C}$ and $5 \% \mathrm{CO}_{2}$.

3. On day 33 , replace medium by freshly prepared DA neurons differentiation medium supplemented with small molecules and growth factors (without Y-27632). Change medium manually every 3-4 days until day 65.

\section{Immunostaining, automated high-throughput image acquisition and analysis}

\section{Fluorescence staining}

1. Perform fluorescent immunostaining as described in the Supplementary File 1: step 4.

2. Image cells as described in the Supplementary File 1: step 1.2.

3. Upload imaging files generated by the imaging system to the image analysis software 2 (see Table of Materials) for further image analysis, as described in step 6.2.

\section{Image analysis using the image analysis software 2}

1. Once imaging files are uploaded in image analysis software 2, go to the "image analysis" function and build the analysis algorithm by using the drop down menu.

2. Select nuclei using the task "find nuclei", which detects regions on the image belonging to cell nuclei (here stained with Hoechst). Exclude nuclei from 
dead cells (pyknotic nuclei) by setting a threshold for nuclear area or diameter.

3. Select the cell cytoplasm using the task "find cytoplasm". This task detects regions around nuclei belonging to cell cytoplasm. Include only wellsegmented cells. Exclude mitotic, apoptotic and badly segmented cells. Remove cells touching the border of the image.

4. Add the tasks "calculate morphology properties" and "calculate intensity properties". The morphology parameters include the calculation of morphology properties such as area for a region of interest. The intensity parameters include the calculation of intensity properties such as mean intensity for a region of interest (e.g. cytoplasm of neurons).

5. Select a subpopulation (e.g., TH positive neurons) of the input population (all cytoplasms selected) using one or more conditions, morphology and/or intensity. NOTE: Usually, intensity is chosen for neurons. Based on negative controls, it is possible to define above which threshold of intensity the neurons are considered positive for $\mathrm{TH}$.

6. Define the output results. This is the last building block of each analysis. It defines the assay readout values for each well of a culture plate (results per well).

7. Run the batch analysis and export the results. Normalize the number of positive cells to the total number of nuclei and represent data as the percentage of positive cells.

\section{Quantitative real-time polymerase chain reaction (qRT-PCR)}

1. Perform the qRT-PCR protocol as described in the Supplementary File 1: step 3.

\section{Representative Results}

Our automated cell culture and imaging system was designed to minimize human intervention allowing us to standardize the cultivation of hiPSC and differentiation into different cell types such as cortical or midbrain dopaminergic (mDA) neurons. A schematic overview of our automated cell culture system with integrated imaging devices is depicted in Figure 1. The initial introduction of cell cultures to this automated cell culture system can either be done by automatically seeding cells from a $50 \mathrm{~mL}$ tube or by using the "Loading Of Culture Plates" or "Loading Of Assay Plates" method for import of culture or assay plates. A central component of our system is the liquid handling station where all liquid transfer steps such as media changes or subcultivations are carried out. The custom-made deck layout of the liquid handler is represented in Figure 2. The liquid handling station is equipped with four positions. Up to four plates can be transferred from the incubator to the deck, allowing parallel media changes. Since in the subcultivation method, both the parent and daughter culture plates have to be accommodated on the deck, the maximum number of culture plates processed in parallel is limited to two. An important feature of the liquid handling station is the possibility to tilt the plates during media change for complete removal of cell culture supernatant. Also, the liquid handling station is equipped with shakers for favoring enzymatic dissociation of cells during the execution of the subcultivation protocol. Our automated culture system is also equipped with two imaging systems: a brightfield imaging cytometer for performing cell counting and confluency checks 
and, therefore, monitoring the cell growth over time, and a dual spinning disk confocal microscope for rapid, high content and high-resolution imaging of cells.

The hiPSC cultures are monitored daily for growth at the brightfield imaging cytometer and analyzed for percentage of confluency. The brightfield image in the left panel and in the right panel a green mask from the analysis of the brightfield image obtained with the cytometer (Figure 3A). A homogeneous hiPSC growth is observed over the time, as shown by the confluence percentages of two hiPSC lines ( $n=4$ plates) grown in parallel and subjected to confluency checks from day 1 to day 6 (Figure 3B). Upon reaching the set threshold, the hiPSC are passaged. The cell lines were cultured manually $(\mathrm{m})$ or by the automation (a) system and observed for maintenance of typical stem cell morphology for at least two passages, representative brightfield images (Figure 4A). The hiPSC cultured manually (not shown) or in the automated system exhibited the typical stem cell marker OCT4 (red) and SSEA4 (green), as shown in the immunofluorescence assay (Figure 4B). The expression of the pluripotency markers OCT4, NANOG and REX1 were also assessed at mRNA level by qRT-PCR (Figure 4C). Relative quantifications were performed with samples collected from one cell line grown manually $(\mathrm{m})$ and in the automated culture system (a) in duplicates (replicates 1 and 2). The expression levels of all three pluripotency markers in the replicates cultivated in the automated culture system are similar to marker expression after manual culture. On day 8 (D8), the expression of pluripotency markers was absent in cortical neurons differentiated (Diff) from hiPSC.

One important application of the automated culture system is the differentiation of hiPSC into different cell types including neurons. Here we show the differentiation of hiPSC into neurons using the NGN2 strategy, which produces a pure cortical neuron culture in a very short time (approximately 6 days). Neurons differentiated in the automated culture system (a) presented similar morphology and neuronal network organization as the neurons cultivated manually $(\mathrm{m})$ (Figure 5A). Automated differentiated cortical neurons were positive for TUBB3 (neuron-specific Class III $\beta$-tubulin, red) and BRN2 (upper cortical layer marker, green) (Figure 5B), comparable to manually differentiated neurons (data not shown). The expression of neuronal markers including the microtubule-associated protein $2(M A P 2)$, the neural cell adhesion molecule (NCAM1) and Synapsin-1 (SYN1), as well as the cortical neuron markers BRN2 and CUX1 (upper cortical layer) were enriched in neurons at day 8 (D8) of differentiation (Figure 5C). Very low or no expression of these markers was observed in hiPSC. Relative quantifications were performed with samples collected from one cell line grown manually $(\mathrm{m})$ and in the automated culture system (a) in duplicates (replicates 1 and 2). The expression levels in replicates show similar variations between manually and automated differentiations.

The integrated imaging capability of the automated culture system allows hands-free data collection for the health of cultures thus enabling long-term automated acquisition of phenotypic readouts. Using the NGN2 approach to a small molecule derived neural precursor (smNPC) line transduced with GFP lentivirus, we established a live-cell automated neurite outgrowth assay in which neurite length was measured over 11 days of differentiation without any manual intervention. The neurite complexity increased over time, as demonstrated by the area occupied with neurites on day 1, 3 and 11, GFP expression and masked images from analysis (Figure 6A, B). The increase in neurite length from day 1 to 11 of differentiation was quantified and showed a 
similar development across different wells. For the sake of simplicity, data from only 3 columns with 6 wells each from a 96-well plate is depicted in the representative graph although all inner 60 wells were analyzed (Figure 6C).

Another application of the automated culture system shown here is the differentiation of hiPSC into mDA neurons. The differentiation is based on media changes following a preestablished protocol and was performed on the automated culture system from days 0 to 65 . Automated media changes did not cause cell detachment or any other visually detectable changes in the differentiation. At the end of the differentiation, on day 65, mDA neurons show cellular organization and morphology (spheric soma, long and spiny dendrites) comparable to manual differentiation (Figure 7A, B). At the mRNA level, mDA neurons differentiated in the automated culture system show the expression of neuronal and mDA markers, MAP2 and TH (tyrosine hydroxylase), respectively (Figure 7C). Both differentiations generated substantial amounts of TH and MAP2 positive neurons (Figure 7D).

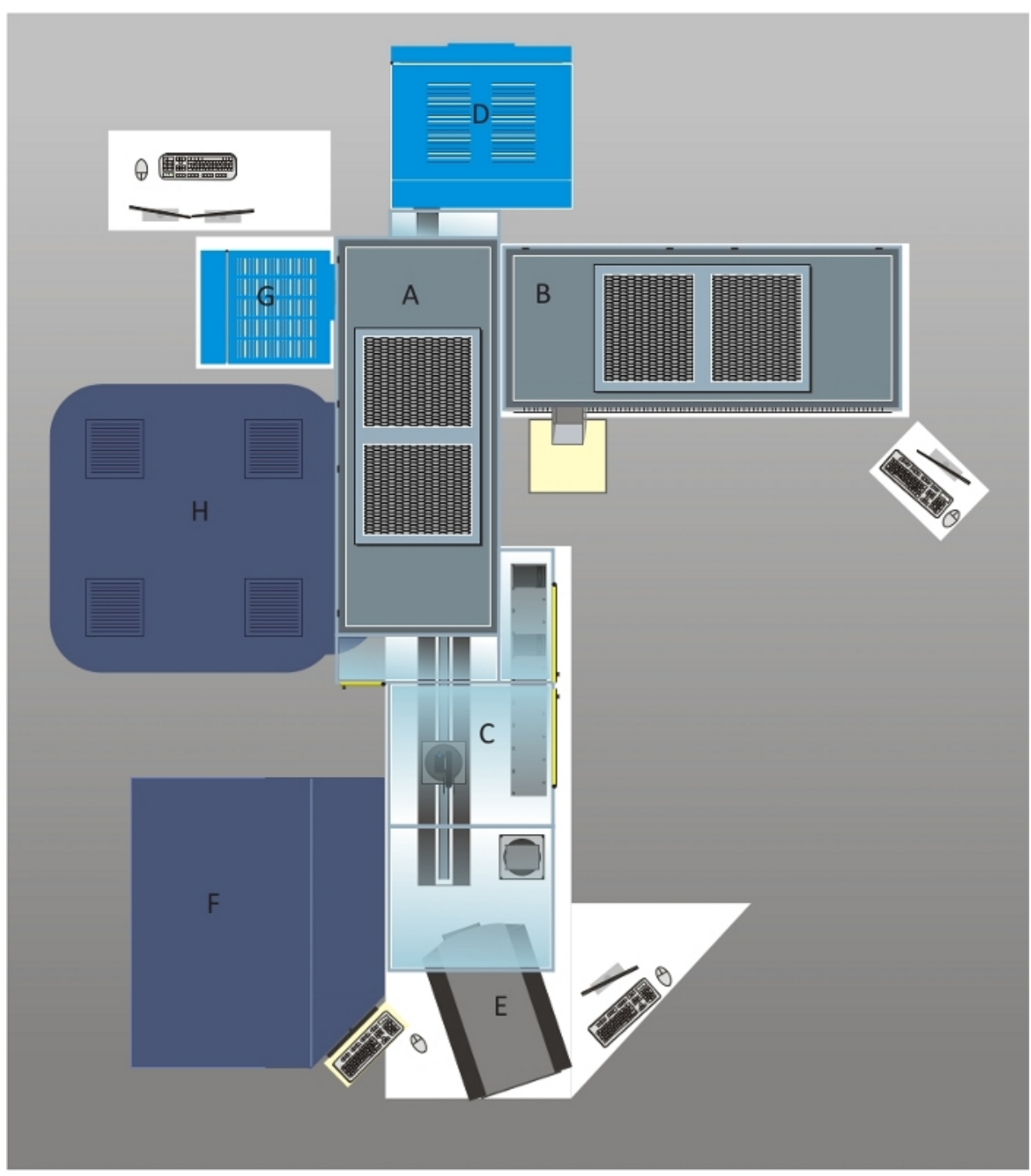

Figure 1: Schematic overview of the automated cell culture and imaging platform. 
The system was designed with a polycarbonate housing and two HEPA hoods ( $A$ and B) equipped with four UV lamps ensuring a sterile environment for cell culture applications. Cell culture plates are loaded on shelves in front of the robotic arm which can be accessed via the front door (C). The plates are loaded into the $\mathrm{CO}_{2}$ incubator (D) with a capacity of 456 plates. A brightfield cell cytometer $(E)$ is used for confluency checks and cell counting during subcultivation routines. The liquid handling station is below one of the HEPA hoods (B). The deck layout of the liquid handler is described in Figure 2. The pipetting arm of the liquid handling station carries a 96 channel pipetting head, eight $1 \mathrm{~mL}$ pipetting channels and four $5 \mathrm{~mL}$ pipetting channels. In the case of the $1 \mathrm{~mL}$ pipetting channels, tips or needles can be used for liquid transfers. For screening purposes, cells seeded in assay plates can be treated with samples stored at $-20^{\circ} \mathrm{C}$ in the automated $-20^{\circ} \mathrm{C}$ storage system $(\mathrm{F})$ after thawing these samples in a second incubator (G). High throughput imaging is performed in the automated confocal microscope $(\mathrm{H})$ offering to acquire images in confocal mode using two spinning discs or in epifluorescence mode. A live-cell chamber integrated into the microscope allows performing long-term imaging of cultured cells. Please click here to view a larger version of this figure. 


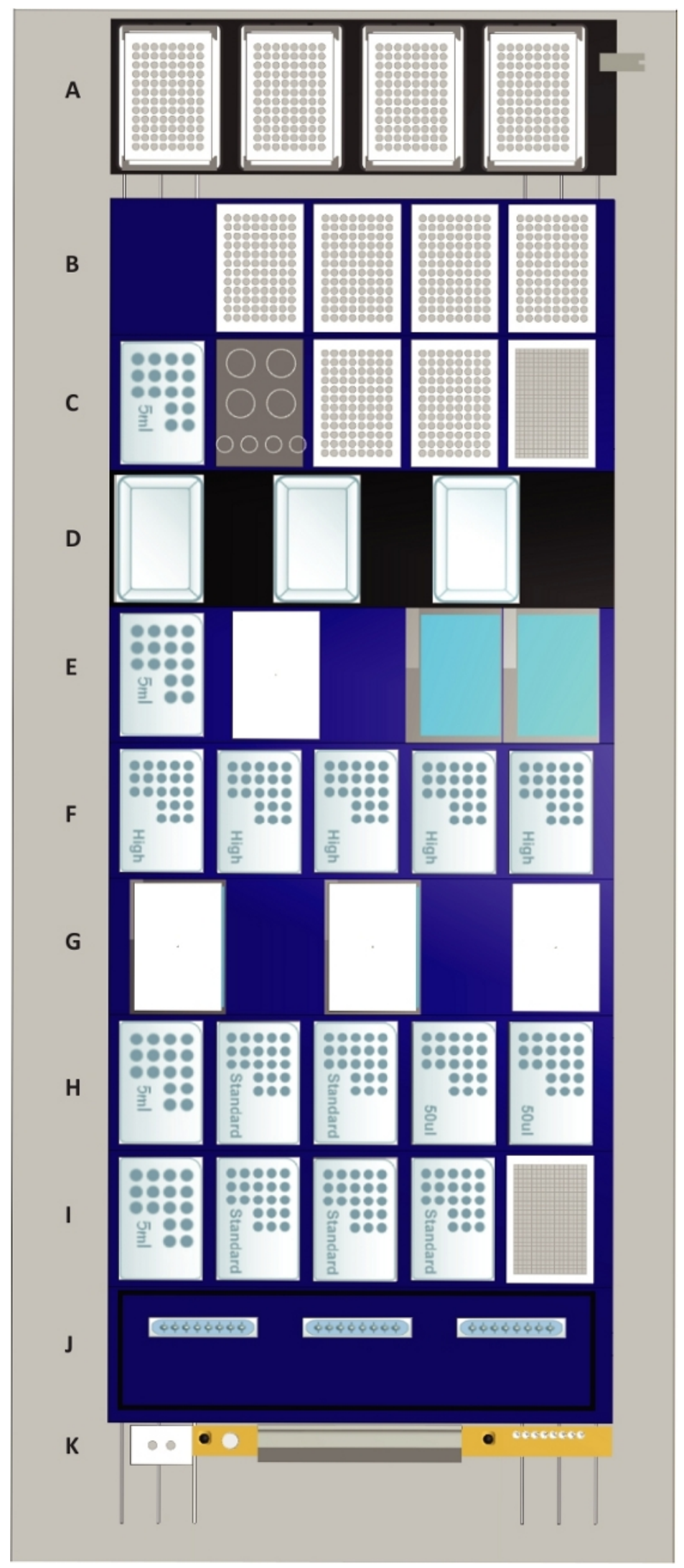


Figure 2: The deck layout of the liquid handling station.

Tip positions are indicated by " $50 \mu \mathrm{L}$ " for $50 \mu \mathrm{L}$ tips, by "standard" for $300 \mu \mathrm{L}$ tips, by "high" for $1 \mathrm{~mL}$ tips and by " $5 \mathrm{~mL}$ " for 5 $\mathrm{mL}$ tips. Deck components: (A) Four heated shaker positions (max speed: $2500 \mathrm{rpm}$ ) which can be used for any culture plate or assay plate format. The shaker positions are equipped with clampable grippers which are also used for plate alignment following transports to the deck. Furthermore, the shaker positions function as a lid parking position for plates during liquid transfer steps. For representative purposes, all shaker positions are occupied by 96 well assay plates. (B) Four tilt modules for processing four plates of any format simultaneously are positioned on the top. The lowest position marks the waste collection chamber for culture and assay plates and the 96 channel pipetting head. For representative purposes, all tilt modules are occupied by 96 -well assay plates. (C) On the top position, the 384-well plate for cell counting is located. Below are two positions for plates that are occupied by 96-well plates for representative purposes and a rack for four $50 \mathrm{~mL}$ and four $15 \mathrm{~mL}$ tubes. The lowest position is occupied by $5 \mathrm{~mL}$ tips. (D) Three media lines with positions for media reservoirs. The media lines possess liquid level sensors that allow to automatically fill the media reservoir with up to $250 \mathrm{~mL}$ of media. (E) Two liquid waste modules with active drain are based on the top, below a temperature-controlled module with a position for one container (white) and a $5 \mathrm{~mL}$ tip rack are located. (F) Five positions for $1 \mathrm{~mL}$ tips. (G) Two temperature-controlled modules are positioned at the bottom and a position for parking one of their lids on the top. $(\mathbf{H})$ Positions for two $50 \mu \mathrm{L}$ nested tip racks (NTR) in the top followed by two positions for single-channel and 96-channel pick up of $300 \mu \mathrm{L}$ tips and a 5 $\mathrm{mL}$ tip rack at the bottom. (I) A stacker for 384-well counting plates at the top followed by three $300 \mu \mathrm{L}$ NTR and a $5 \mathrm{~mL}$ tip rack at the bottom. (J) Storage and wash station for three sets of eight reusable metal $1 \mathrm{~mL}$ needles. (K) Waste position for $1 \mathrm{~mL}$ and $5 \mathrm{~mL}$ pipetting channels and empty NTR (grey) as well as a gripper block for 1 and $5 \mathrm{~mL}$ channels (white) used for on-deck transport steps. Please click here to view a larger version of this figure. 

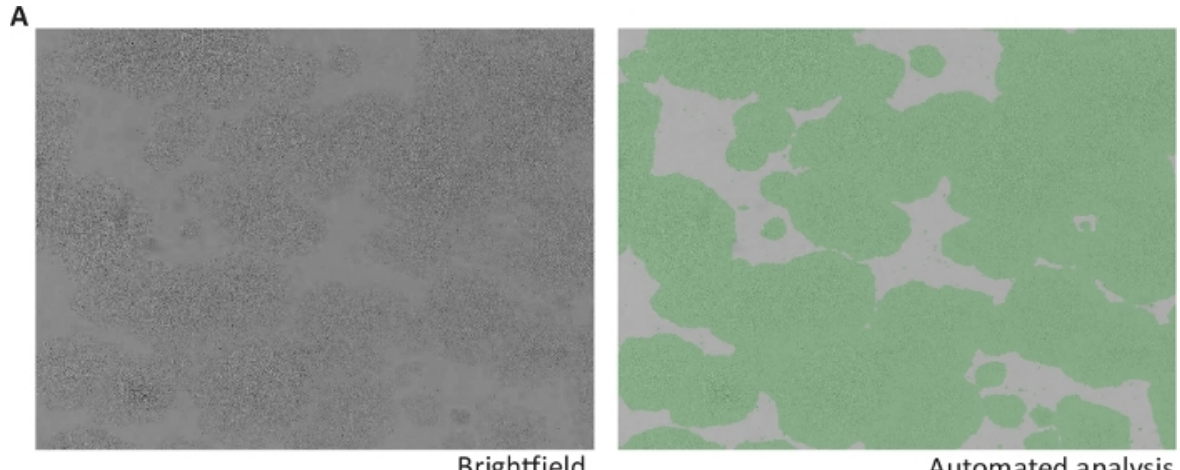

Automated analysis

B

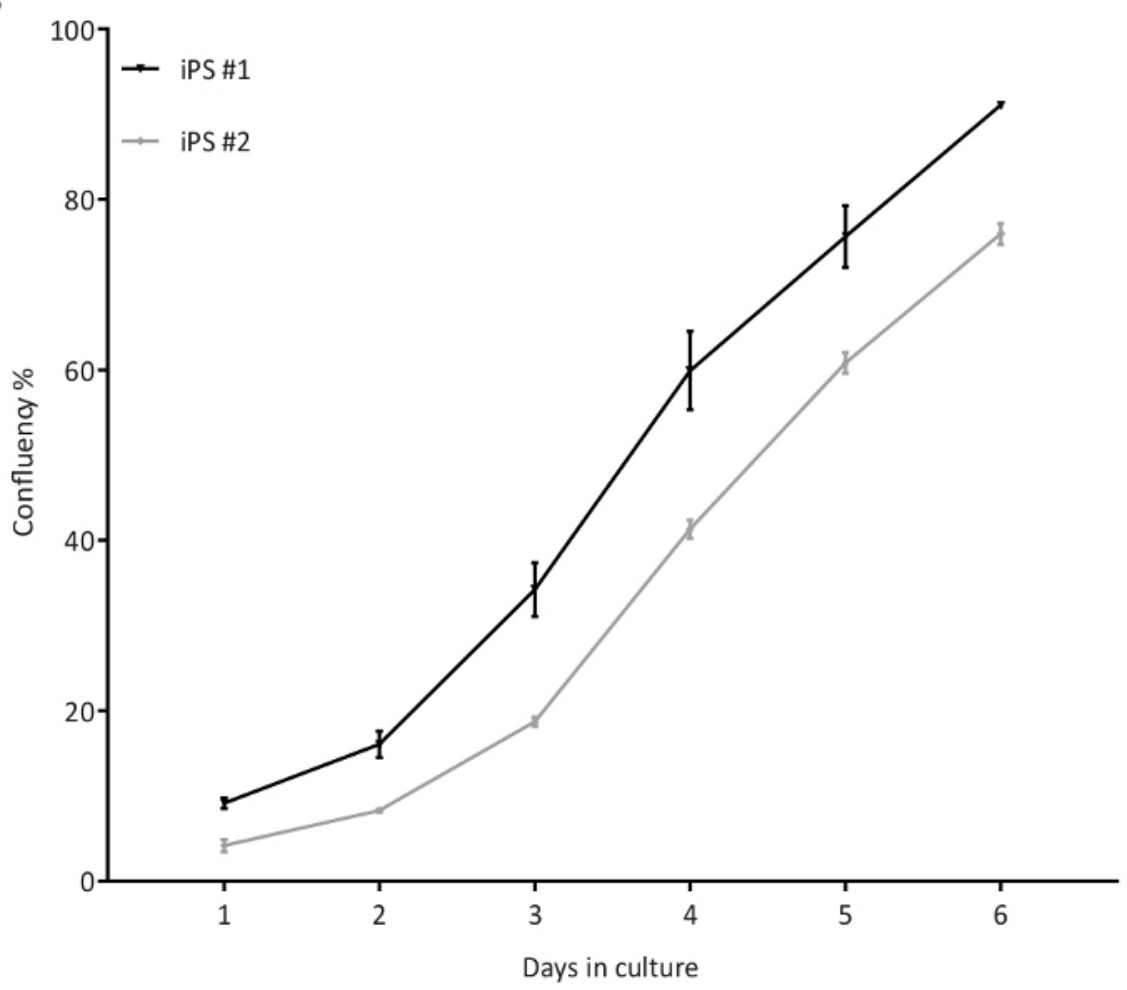

Figure 3: Automated confluency check of hiPSC.

(A) Representative brightfield (BF) images of hiPSC taken by the cell cytometer (left) and after automated confluency analysis (right) indicating the proportional area occupied by cells in green; (B) Confluency percentages recorded from two hiPSC lines (iPS \#1 and \#2) from day 1 to 6 of culture, $n=41$-well plates per cell line. Please click here to view a larger version of this figure. 


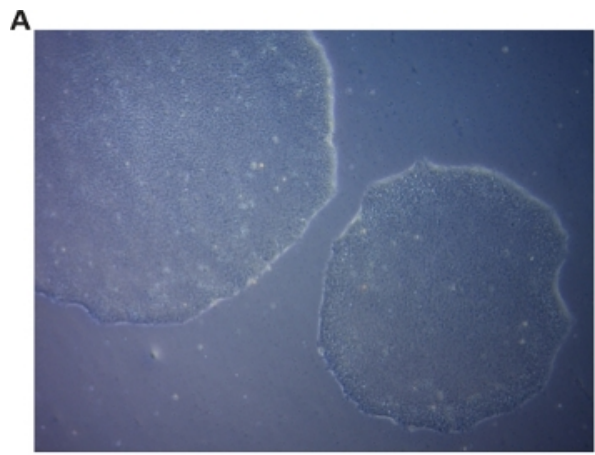

Manual (m)

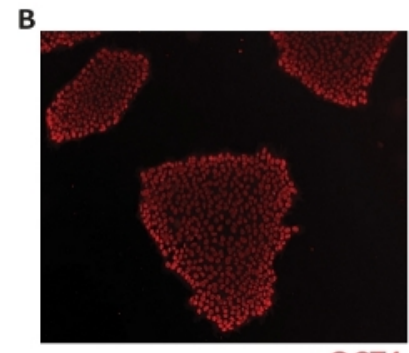

OCT4

C

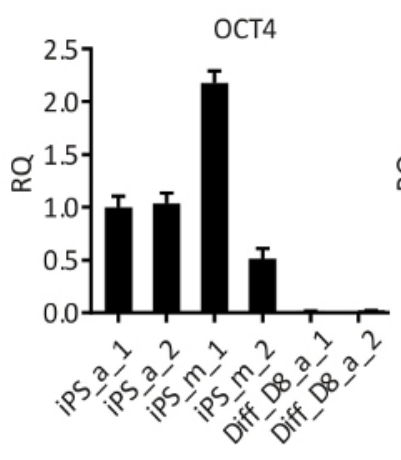

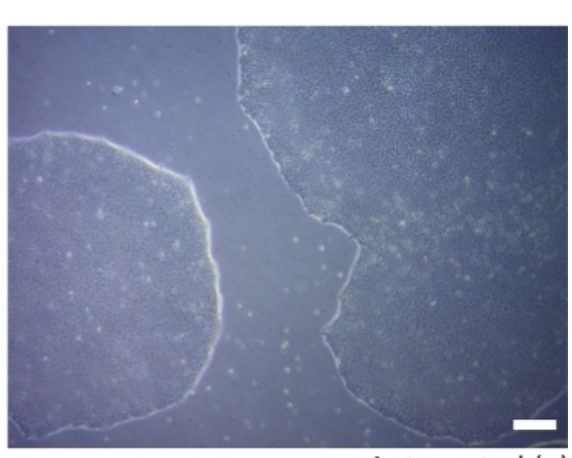

Automated (a)
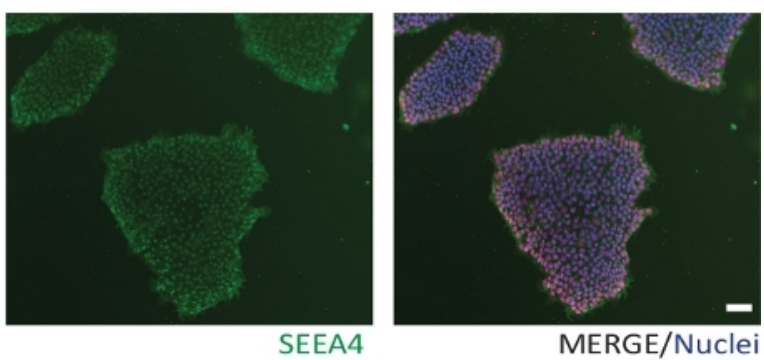

SEEA4
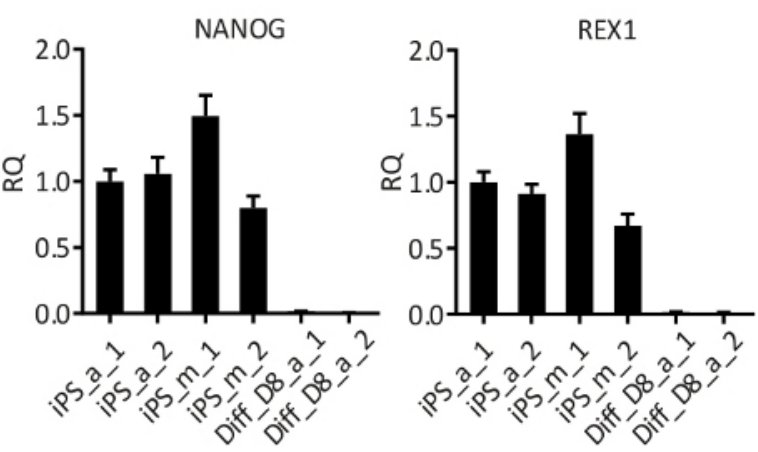

\section{Figure 4: Passaging of hiPSC.}

(A) BF image of one hiPSC line grown manually (left) and using the automated culture system (right). Images were taken 6 days after the second passaging; (B) Representative images of hiPSC stained for the pluripotency markers OCT4 and SSEA4, and counterstained with Hoechst 33342 (Nuclei); (C) Results of qRT-PCR for the pluripotency markers OCT4, NANOG and REX1 in one hiPSC line cultivated in duplicates (1 and 2 ) manually $(\mathrm{m})$ and in the automated culture system (a), and the respective hiPSC-derived cortical neurons (Diff) at day 8 (D8) of differentiation. The data is represented as the relative quantity (RQ) using iPS_a_1 as the reference sample. Error bars represent standard deviation (SD) from 3 technical replicates of qRT-PCR reaction. GAPDH, RPL13A1 and RPLPO were used as housekeeping genes. Scale bar: $100 \mu \mathrm{m}$. 


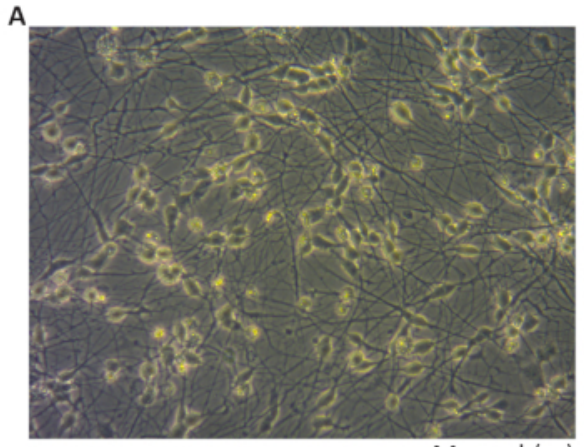

Manual (m)

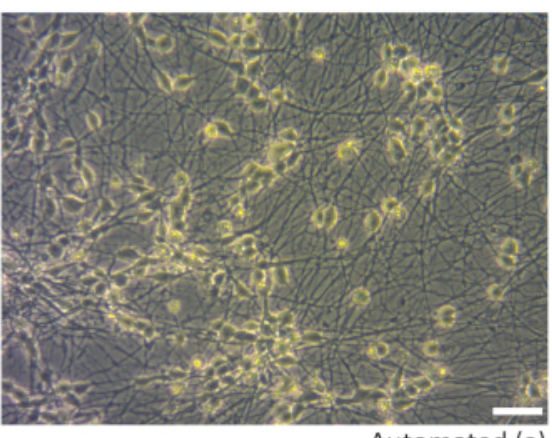

Automated (a)
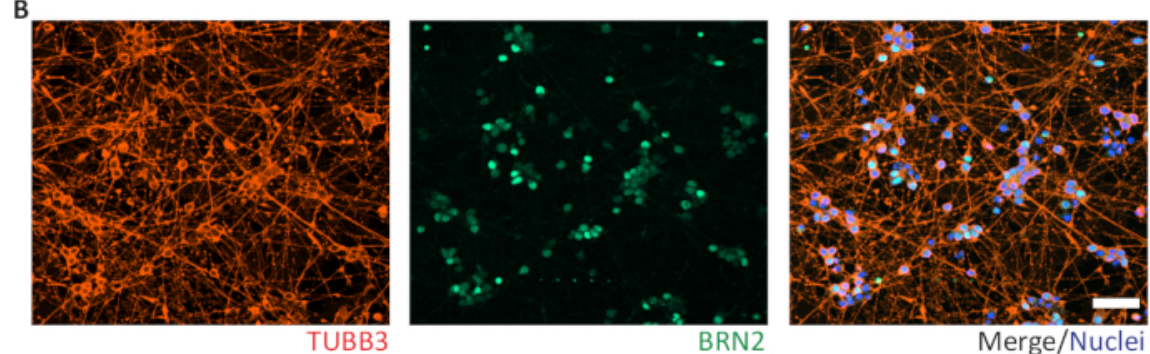

C
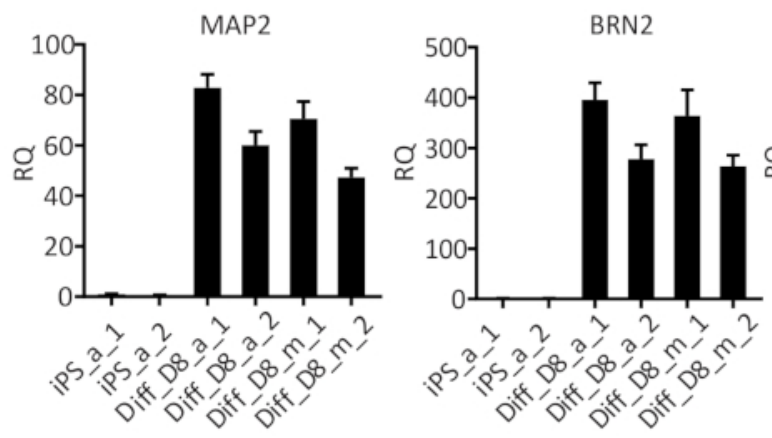

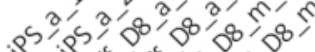

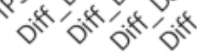
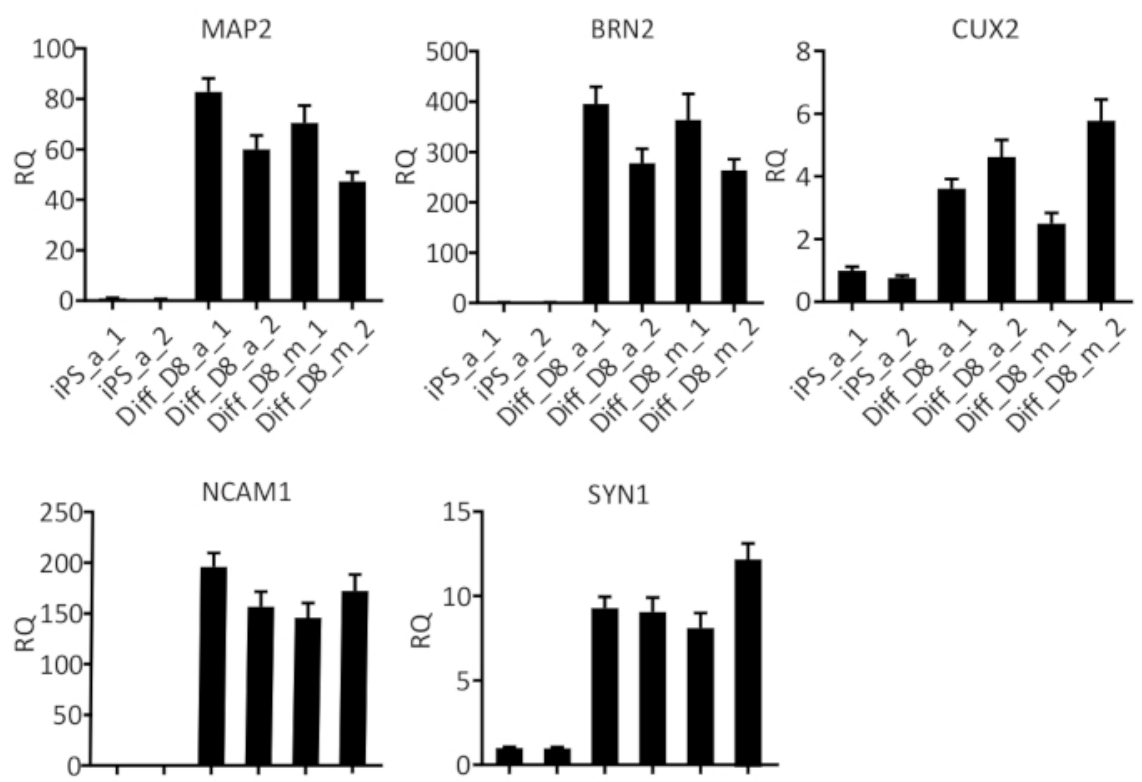

$>2 \geq 22^{2}$ $8,5,2,80,8,8,8,5$

ind 0 ind

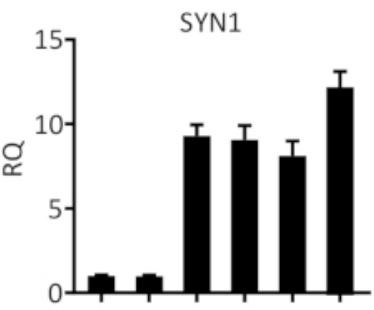

$\geq 2 \geq 22^{2}$

$8,5,8,8,8,8,8$,

Figure 5: Human iPSC-derived cortical neurons. 
(A) Brightfield images of day 6, manually $(\mathrm{m})$ and automated (a) differentiated cortical neurons showing similar neuronal networks; (B) Representative images of cells stained for TUBB3 (pan neuronal), BRN2 (cortical neurons) and Hoechst 33342 (nuclei) on day 8 of differentiation; (C) Results of qRT-PCR for marker genes of cortical neurons (MAP2, BRN2, CUX2, NCAM1 and SYN1) enriched in day 8 (D8) of differentiation. The data is represented as the relative quantity (RQ) using iPS_a_1 as the reference sample. Error bars represent the SD from 3 technical replicates of qRT-PCR reaction. GAPDH, RPL13A1 and RPLPO were used as housekeeping genes. Scale bar: $50 \mu \mathrm{m}$. Please click here to view a larger version of this figure. 


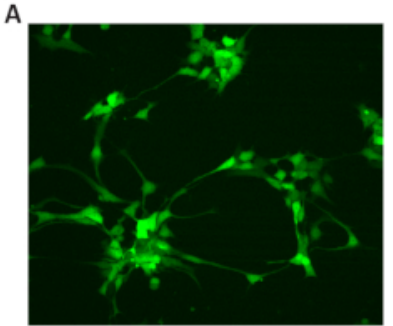

Day1

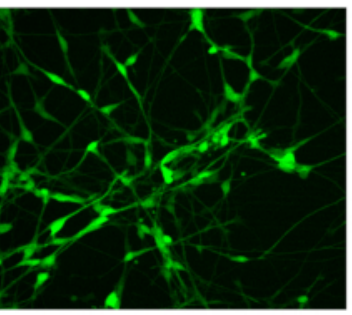

Day3

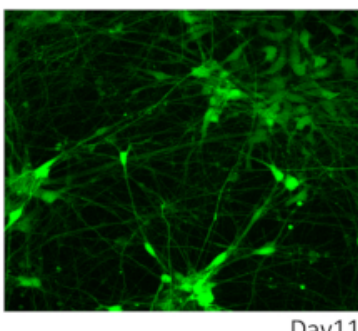

Day11

B

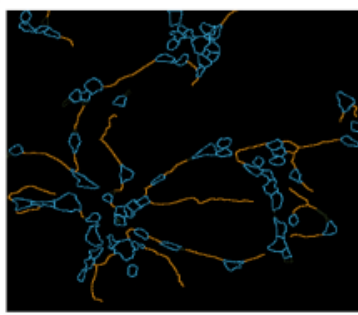

Day1

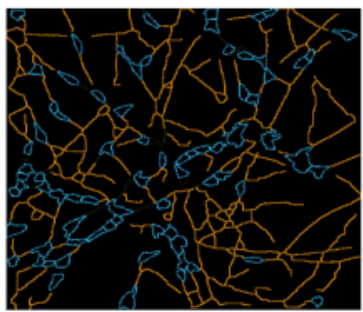

Day3

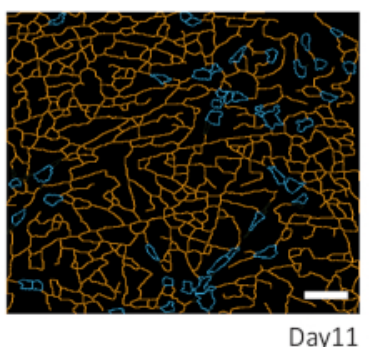

C

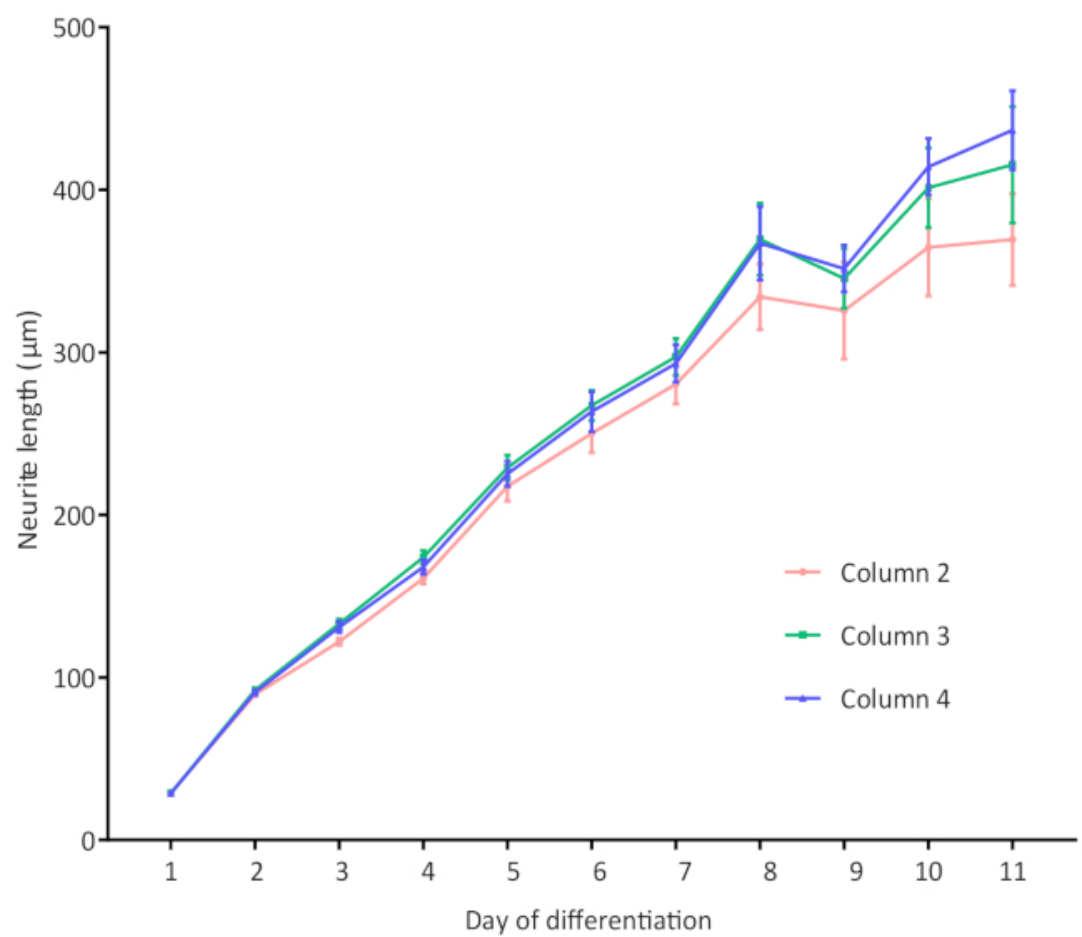

Figure 6: A high-throughput assay for neurite outgrowth.

(A) Representative images of GFP expressing cells on days 1, 3 and 11 of differentiation; (B) Representative binary images of neurites on days 1, 3 and 11 of differentiation. The neurite outgrowth was quantified using the high content image analysis software 1 and is represented as neurite length; (B) The graphic displays the increase in neurite length in NPC-derived 
NGN2 neurons and formation of a dense network. Three 96 -well plates with cells in the inner 60 wells are imaged. For simplicity, only three columns of wells per 96 -well plate are shown as an example with $n=6$ wells per column. An average of 1308 cells were analyzed per well. Error bars represent the standard error of the mean (S.E.M.). Scale bar $=50 \mu \mathrm{m}$. Please
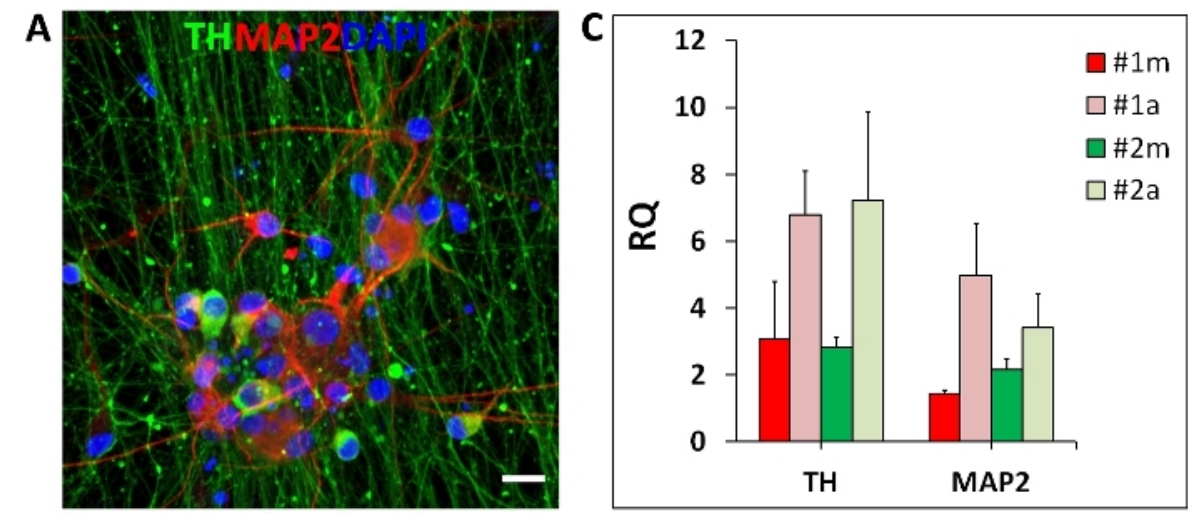

B
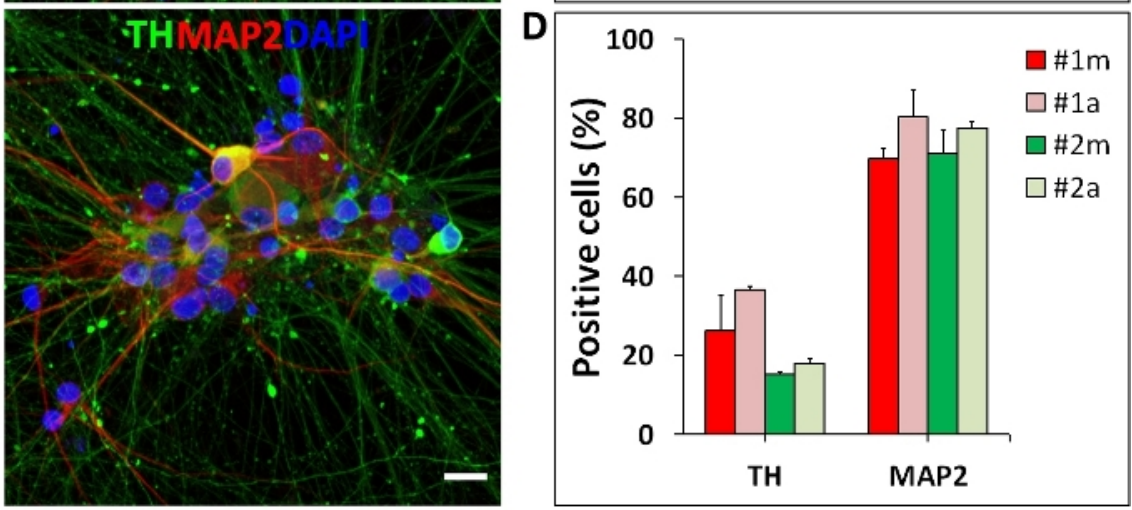

\section{Figure 7: Human iPSC-derived mDA neurons.}

Midbrain DA neurons differentiated manually $(\mathrm{m})$ and in the automated (a) culture system. (A, B) Representative fluorescent images of mDA neurons stained for tyrosine hydroxylase (TH, mDA neuron marker; green), MAP2 (neuronal marker; red) and Hoechst 33342 (nuclei; blue); (C) Representative qRT-PCR results for marker genes of mDA neurons differentiated manually and in the automated culture system. $T H$ and $M A P 2$ expression levels are represented as relative quantity (RQ) normalized to housekeeping genes (OAZ1 and GAPDH); (D) Percentages of TH and MAP2 positive neurons generated by manual and automated differentiation. Error bars represent the SD of two independent differentiations performed with two distinct iPSC lines (\#1 and \#2). Scale bar = $50 \mu \mathrm{m}$. Please click here to view a larger version of this figure. 


\begin{tabular}{|c|c|c|c|c|c|}
\hline Cell type & Purpose & Protocol step & Cell density & Plate format & Cell number/well \\
\hline \multicolumn{6}{|l|}{$\begin{array}{c}\text { NGN2 } \\
\text { differentiation }\end{array}$} \\
\hline iPSC & $\begin{array}{l}\text { NGN2 stable } \\
\text { line generation }\end{array}$ & S.2.3. & $30,000 \mathrm{cells} / \mathrm{cm}^{2}$ & 12-well & $1,17,000$ \\
\hline iPSC & $\begin{array}{l}\text { NGN2 neuron } \\
\text { differentiation }\end{array}$ & 3.1.3. & 30,000 cells $/ \mathrm{cm}^{2}$ & 1-well & $25,20,000$ \\
\hline iPSC & $\begin{array}{l}\text { NGN2 neuron } \\
\text { differentiation }\end{array}$ & 3.1.3. & 30,000 cells $/ \mathrm{cm}^{2}$ & 96-well & 9,600 \\
\hline \multicolumn{6}{|l|}{$\begin{array}{c}\text { smNPC } \\
\text { generation }\end{array}$} \\
\hline smNPC & $\begin{array}{l}\text { Replating day } \\
12 \text { and } 16\end{array}$ & S5.9. and S5.11. & 70,000 cells $/ \mathrm{cm}^{2}$ & 6-well & $6,72,000$ \\
\hline smNPC & $\begin{array}{c}\text { Replating from } \\
\text { passage } 5\end{array}$ & 5.11. & 50,000 cells $/ \mathrm{cm}^{2}$ & 6-well & $4,80,000$ \\
\hline smNPC & $\begin{array}{c}\text { smNPC to } \\
\text { NGN2 neurons }\end{array}$ & 3.2 .2 & $50,000 \mathrm{cells} / \mathrm{cm}^{2}$ & 96-well & 16,000 \\
\hline \multicolumn{6}{|l|}{$\begin{array}{c}\text { mDA } \\
\text { differentiation }\end{array}$} \\
\hline iPSC & $\begin{array}{l}\text { mDA neuron } \\
\text { differentiation }\end{array}$ & 4.1.2. & 200,000 cells $/ \mathrm{cm}^{2}$ & 1-well & $1,68,00,000$ \\
\hline DA neurons & Day 25 replating & 4.3.2. & $400,000 \mathrm{cells} / \mathrm{cm}^{2}$ & 1-well & $3,36,00,000$ \\
\hline DA neurons & Day 25 replating & 4.5.2. & 100,000 cells $/ \mathrm{cm}^{2}$ & 96-well & 32,000 \\
\hline Coating & Purpose & Protocol step & $\begin{array}{c}\text { Concentration/ } \\
\text { Dilution }\end{array}$ & Plate format & Details of coating \\
\hline \multicolumn{6}{|l|}{ iPS culture } \\
\hline Extracellular matrix & $\begin{array}{c}\text { ¡PSC, NGN2 line, } \\
\text { mDA neurons }\end{array}$ & 1.5.7. and S2.3. & $\begin{array}{c}1 \text { aliquot*; } \\
25 \mathrm{~mL} \text { DMEM/F-12 }\end{array}$ & 1-/12-well & $\begin{array}{c}8 / 0.5 \mathrm{~mL} / \\
\text { well; } 1 \mathrm{~h} \text { at RT }\end{array}$ \\
\hline $\begin{array}{c}\text { NGN2 } \\
\text { differentiation }\end{array}$ & & & & & \\
\hline
\end{tabular}




\begin{tabular}{|c|c|c|c|c|c|}
\hline Poly-L-Ornithine & $\begin{array}{l}\text { NGN2 neuron } \\
\text { differentiation }\end{array}$ & 3.1.3. and 3.2.2. & $0.1 \mathrm{mg} / \mathrm{mL} ; \mathrm{PBS}$ & 1-/96-well & $\begin{array}{l}\text { 8/0.1 mL/well; } \\
12 \mathrm{~h} \text { at } 37^{\circ} \mathrm{C} ; \\
3 x \text { PBS wash }\end{array}$ \\
\hline Laminin & $\begin{array}{l}\text { NGN2 neuron } \\
\text { differentiation }\end{array}$ & 3.1.3. and 3.2.2. & $5 \mu \mathrm{g} / \mathrm{mL} ;$ PBS & 1-/96-well & $\begin{array}{c}8 / 0.1 \mathrm{~mL} / \text { well; } \\
4 \mathrm{~h} \text { at } 37^{\circ} \mathrm{C}\end{array}$ \\
\hline \multicolumn{6}{|l|}{$\begin{array}{c}\text { smNPC } \\
\text { generation }\end{array}$} \\
\hline Extracellular matrix & $\begin{array}{c}\text { smNPC generation } \\
\text { and culture }\end{array}$ & $\begin{array}{l}\text { S5.6., S5.9., } \\
\text { S5.11. }\end{array}$ & $\begin{array}{c}1 \text { aliquot*}^{*} ; \\
25 \text { mL DMEM/F-12 }\end{array}$ & 6-well & $1 \mathrm{~mL} ; 2 \mathrm{~h}$ at $\mathrm{RT}$ \\
\hline \multicolumn{6}{|l|}{$\begin{array}{c}\text { mDA } \\
\text { differentiation }\end{array}$} \\
\hline Extracellular matrix & mDA differentiation & 4.1.2. & $\begin{array}{c}1 \text { aliquot }^{*} ; \\
25 \text { mL DMEM/F-12 }\end{array}$ & 1-well & $\begin{array}{l}12 \mathrm{~mL} ; 12 \\
\text { h at } 37^{\circ} \mathrm{C}\end{array}$ \\
\hline Poly-L-Ornithine & mDA differentiation & 4.3.2. and 4.5.2. & $0.1 \mathrm{mg} / \mathrm{mL} ;$ PBS & 1-/96-well & $\begin{array}{l}12 / 0.1 \mathrm{~mL} / \text { well; } \\
12 \mathrm{~h} \text { at } 37^{\circ} \mathrm{C} ; \\
3 x \text { PBS wash }\end{array}$ \\
\hline Laminin & mDA differentiation & 4.3.2. and 4.5.2. & $10 \mu \mathrm{g} / \mathrm{mL} ;$ PBS & 1-/96-well & $\begin{array}{c}12 / 0.1 \mathrm{~mL} / \text { well; } \\
12 \mathrm{~h} \text { at } 37^{\circ} \mathrm{C}\end{array}$ \\
\hline Fibronectin & mDA differentiation & 4.3.2. and 4.5.2. & $2 \mu \mathrm{g} / \mathrm{mL} ;$ PBS & 1-/96-well & $\begin{array}{c}12 / 0.1 \mathrm{~mL} / \text { well; } \\
12 \mathrm{~h} \text { at } 37^{\circ} \mathrm{C}\end{array}$ \\
\hline
\end{tabular}

${ }^{*}$ A extracellular matrix aliquot is defined as the dilution factor (in $\mu \mathrm{L}$ ) present in the Certificate of Analysis of this product.

Table 1: Seeding cell density and coating respective to the plate format. 


\begin{tabular}{|c|c|}
\hline Day & Reagent \\
\hline Day $0-1$ & 100 nM LDN193189, 10 нM SB431542 \\
\hline Day $1-3$ & $\begin{array}{c}100 \text { nM LDN193189, } 10 \text { M SB431542, } 1 \text { mM SHH, } 2 \text { mM Purmorphamine, } \\
\text { 100ng/mL FGF-8b }\end{array}$ \\
\hline Day $3-5$ & 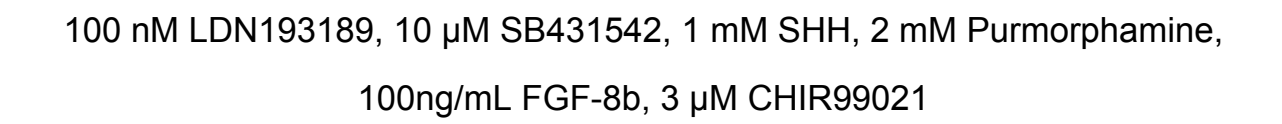 \\
\hline Day $5-7$ & $\begin{array}{c}100 \text { nM LDN193189, } 1 \text { mM SHH, } 2 \text { mM Purmorphamine, 100ng/mLFGF-8b, } \\
3 \mu \mathrm{M} \text { CHIR99021 }\end{array}$ \\
\hline Day 7 - 9 & 100 nM LDN193189, 1 mM SHH, 3 нM CHIR99021 \\
\hline Day $9-11$ & 100 nM LDN193189, 1 mM SHH, 3 нM CHIR99021 \\
\hline Day $11-13$ & $\begin{array}{c}3 \mu \mathrm{M} \text { CHIR99021, } 20 \mathrm{ng} / \mathrm{mL} \text { BDNF, } 0.2 \mathrm{mM} \text { L-ascorbic acid (AA1), } 20 \mathrm{ng} / \mathrm{mL} \\
\text { GDNF, } 1 \mathrm{mM} \mathrm{db-CAMP,} 1 \mathrm{ng} / \mathrm{mL} \text { TGFß3, } 10 \mu \mathrm{M} \text { DAPT }\end{array}$ \\
\hline Day $13-65$ & $\begin{array}{c}20 \mathrm{ng} / \mathrm{mL} \text { BDNF, } 0.2 \mathrm{mM} \text { L-ascorbic acid (AA1), } 20 \mathrm{ng} / \mathrm{mL} \text { GDNF, } 1 \mathrm{mM} \text { db-cAMP, } \\
1 \mathrm{ng} / \mathrm{mL} \text { TGFß3, } 10 \mu \mathrm{M} \text { DAPT }\end{array}$ \\
\hline
\end{tabular}

Table 2: Small molecule addition for dopaminergic neuron differentiation.

\begin{tabular}{|c|c|c|c|}
\hline Day & KSR medium & N2 medium & Differentiation medium \\
\hline Day -1 & $100 \%$ & 0 & 0 \\
\hline Day $1-3$ & $100 \%$ & 0 & 0 \\
\hline Day $3-5$ & $100 \%$ & $25 \%$ & 0 \\
\hline Day $5-7$ & $75 \%$ & $50 \%$ & 0 \\
\hline Day $7-9$ & $50 \%$ & $75 \%$ & $100 \%$ \\
\hline Day $9-11$ & $25 \%$ & 0 & $100 \%$ \\
\hline Day $11-13$ & 0 & 0 & 0 \\
\hline Day $13-65$ & 0 & 0 & 0 \\
\hline
\end{tabular}

Table 3: Media gradient for dopaminergic neuron differentiation. 
Supplementary File 1. Please click here to download this file.

\section{Discussion}

We introduce an automated cell culture system with integrated imaging capabilities for the standardization of hiPSC culture and neuronal differentiation. Due to minimal user intervention, experimental variation is low ensuring reproducibility of cellular phenotypes during differentiation. The calendar-based scheduler supports the organization and parallelization of experiments and allows a high degree of flexibility at which time the experiments are carried out. Existing methods can be easily adapted and the spectrum of available methods can be increased. Additionally, a large number of assay plate formats can be used adding to the flexibility of this system. The minimal system consisting of a $\mathrm{CO}_{2}$ incubator, a robotic arm, a brightfield cell cytometer, and a liquid handling station forms the basic unit needed for hiPSC culture and differentiation, with affordable costs to academic research laboratories. The combination of the automated cell culture system with an automated $-20{ }^{\circ} \mathrm{C}$ storage system for storage of compounds, RNAi libraries or CRISPR/Cas9 libraries, and the integration of a highcontent/high-throughput microscope enable the execution of phenotypic screenings.

In the current study, the automated cell culture system used disposable tips and the culture media was refilled manually into the reservoir, thus limiting the use of the liquid handling station for media changes and other culture processes especially overnight. To circumvent this limitation, the methods can be adjusted to needle usage instead of disposable tips and, after installing tube connections between media lines and media bags stored in a fridge, media reservoirs can be automatically refilled with fresh media pre-warmed by heater elements. This would reduce user interferences caused by manual refilling of tips, culture media and reservoir exchanges.

Our automated cell culture system offers several advantages. One is the barcode tracking system. The plates loaded in the system are identified by a unique barcode which is read and saved by the system allowing tracking of samples during and after method execution. Another advantage is the possibility to create user specific projects. Here, culture plates loaded in the system can be assigned to a specific project and grouped in batches. The structuring in batches simplifies the execution of the same procedure to all plates of a certain batch since no individual plates need to be selected. Additionally, a liquid class editor allows to adjust the pipetting speed and height as well as the aspiration and dispensing parameters for each liquid transfer step. Every process is documented in log files allowing to retrace which tasks have been performed for a given culture or assay plate.

Neurons and other cell types derived from human induced pluripotent stem cells (hiPSC) are useful in vitro tools for studying the mechanisms of neurodegenerative diseases in specific patient populations (e.g. dopaminergic neurons for Parkinson's disease) offering the possibility for personalized drug screenings. Culturing hiPSC is very time intensive and demands trained people to execute complex differentiation protocols, usually limited to low scale production. We adapted the feeder-free culture of hiPSC to an automated culture and implemented two neuronal differentiation protocols, a rapid cortical neuron differentiation protocol based on NGN2 over-expression under a teton promoter ${ }^{10,11}$, and a long-term small molecule-based protocol for generation of midbrain dopaminergic (mDA) neurons ${ }^{13}$. The straightforward transfer and reproducibility 
of manual culture and differentiation protocols makes the automated culture system very useful. Human iPSC cultured in the automated cell culture system showed consistent stem cell morphology and expressed important pluripotency markers, reproducible between independent experiments. In addition, the automation of the hiPSC culture protocol favored the culture and expansion of a larger number of cell lines in parallel. Automated confluency checks scheduled to be performed overnight saved time leaving the system free during the day for downstream process steps carried out when the user was in the laboratory (e.g., harvesting of cells or manual replating for differentiations). On reaching the user-defined confluence threshold, cells are passaged and replated into extracellular matrix-coated plates available on the stacker of the automated cell culture system. Each passage round takes about $70 \mathrm{~min}$ and generates four 1-well plates from one parent plate, which translates to a capacity of 20 passages in a day.

The automation of the NGN2 differentiation protocol was done successfully and allowed the generation of a homogeneous population of neuronal cells across different passages and comparable to manual differentiations. Moreover, the experimental costs for large-scale screening studies involving multiple cell lines or screening experiments with thousands of test conditions/compounds would be reduced due to rapid differentiations. Cost-effective and high-throughput readouts including live-cell neurite outgrowth measurements can be easily developed, implemented and used as phenotypic readouts for disease modeling, as shown previously $14,15,16$. Thus, we further adapted the NGN2 protocol using small molecule derived neural precursor (smNPC) cells that constitutively over-express GFP. The smNPC cells offer further advantages including reduced costs with culture media (one third of the cost with iPSC culture) and time required to scale up experiments. The cell yields from smNPC are 7 to 10 times higher than that obtained with iPSC. The differentiating neurons were successfully monitored and imaged for several days using a fully automated imaging process without the need of manual antibody stainings or chemical labeling, saving costs and time required for manual procedures including the imaging by itself. The current imaging of inner 60 wells of a 96-well plate takes around 16 min per plate when 25 fields per well are imaged, which means that the data for an imaging-based screening for 1000 compounds, could be acquired and analyzed in a day. In the future, this readout could be used in compound screening studies for the rescue of neurite outgrowth defects.

Further, we also demonstrate the transfer of a manual differentiation protocol for generating midbrain dopaminergic (mDA) neurons from iPSC. This small molecule-based differentiation protocol takes 65 days and is labor intensive because of the multiple replating steps and frequent media changes, mostly every 2 days, which limits the production of mDA neurons to few iPSC lines at the same time. The automated mDA differentiation protocol has the great advantage of scaling up the differentiation to dozens of iPSC lines. Up to 30 cell lines could be differentiated in parallel. Since the differentiation is mostly based on media changes, almost the whole differentiation process can be conducted without human interference. Using the calendarbased scheduler of the automated system, we could plan the media changes according to the differentiation steps. One limitation of working with such a large number of cell lines and culture plates was the impossibility to perform overnight media changes. The main reason is the fact that our system is set up for using disposable tips and manual refilling of culture media requiring an user in the laboratory to execute this manual step. To facilitate the 
media change process, plates loaded in the system were assigned to a project and grouped in batches. The batch size was then adapted to the number of disposable tips and volume of culture media available. As discussed above, this limitation can be easily overcome by implementation of reusable/washable needles and automated refilling of media. Automated passaging/replating of cells, as shown for iPSC, is one of the conveniences offered by our automated cell culture system. We have tested the automated replating of mDA neurons on day 25 of differentiation. However, the dissociation of mDA neurons requires longer (40 $\mathrm{min}$ ) incubation with the dissociation enzyme than iPSC (8 min) extending the automated replating process to more than $1 \mathrm{~h}$ per cell lines. As a consequence, the automated replating of 30 cell lines in the same day became impossible. Speeding up other steps during automated replating (transport of plates, pipetting) and adapting the system to the use of needles and media line that makes an overnight work possible would resolve this limitation. Despite the drawbacks, we could successfully transfer the manual protocol to an automated differentiation of mDA neurons producing cultures with substantial amounts of MAP2 (neuron) and TH (mDA neurons) positive cells.

Differentiating dozens of iPSC cell lines in parallel is of great interest in projects that investigate the molecular mechanisms of neurodegenerative diseases, including Parkinson's disease. However, to complete tasks faster with fewer errors and at reduced costs is a big challenge. Due to the automation of the protocols presented here (iPSC, smNPC and mDA neuron), we could speed up, reduce the costs and increase reproducibility in our projects. The development of projects like FOUNDIN-PD (https:// www.foundinpd.org/wp/) involving hundreds of patient cell lines shows need for automated culture and differentiation

protocols. Our future perspectives include the transfer of manual 3 dimensional (3D) cell culture models to the automated system. Minor adaptations in the plate definition settings and the use of adaptors will allow the use of commercial or custom-made plates and microfluidic chambers required for the 3D cultures. Moreover, the implementation of an automated label-free imaging model will allow us to track the neuronal growth in real time and translate changes in neurite outgrowth, neuron organization and cell death into better understanding of the disease mechanisms.

\section{Disclosures}

We have nothing to disclose.

\section{Acknowledgments}

The authors gratefully acknowledge the patients and their families who contributed biomaterial for this study. Cells lines used in the study were from NINDS collection with Rutgers (ND41865 as iPS\#1) and the lab of Dr. Tilo Kunath (iPS\#2). This work is supported in part by the NOMIS Foundation (PH), RiMod-FTD, an EU Joint Programme - Neurodegenerative Disease Research (JPND) (PH); The DZNE I2A initiative (AD); PD-Strat, an ERA-Net ERACoSysMed funded project $(\mathrm{PH})$ and the Foundational Data Initiative for Parkinson's Disease (FOUNDIN-PD) (PH, EB). FOUNDIN-PD is part of The Michael J. Fox Foundation's PATH to PD program. The authors thank Steven Finkbeiner and Melanie Cobb (Gladstone Institutes) for contributing to the establishment of the manual mDA neuron differentiation protocol and Mahomi Suzuki (Yokogawa Electric Corporation) for assistance in neurite outgrowth analysis setup.

\section{References}


1. Takahashi, K. et al. Induction of pluripotent stem cells from adult human fibroblasts by defined factors. Cell. 131 (5), 861-872 (2007).

2. Payne, N.L., Sylvain, A., O'Brien, C., Herszfeld, D., Sun, G., Bernard, C.C.A. Application of human induced pluripotent stem cells for modeling and treating neurodegenerative diseases. New biotechnology. 32 (1), 212-228 (2015).

3. Wu, Y.Y., Chiu, F.L., Yeh, C.S., Kuo, H.C. Opportunities and challenges for the use of induced pluripotent stem cells in modelling neurodegenerative disease. Open Biology. 9 (1), 180177 (2019).

4. Avior, Y., Sagi, I., Benvenisty, N. Pluripotent stem cells in disease modelling and drug discovery. Nature Reviews Molecular cell biology. 17 (3), 170-182 (2016).

5. Jain, S., Heutink, P. From single genes to gene networks: high-throughput-high-content screening for neurological disease. Neuron. 68 (2), 207-217 (2010).

6. Soares, F.A.C., Chandra, A., Thomas, R.J., Pedersen, R.A., Vallier, L., Williams, D.J. Investigating the feasibility of scale up and automation of human induced pluripotent stem cells cultured in aggregates in feeder free conditions. Journal of biotechnology. 173, 53-58 (2014).

7. Konagaya, S., Ando, T., Yamauchi, T., Suemori, H., Iwata, H. Long-term maintenance of human induced pluripotent stem cells by automated cell culture system. Scientific reports. 5, 16647 (2015).

8. Conway, M.K. et al. Scalable 96-well Plate Based iPSC Culture and Production Using a Robotic Liquid Handling System. Journal of visualized experiments: JoVE. (99), e52755 (2015).
9. Paull, D. et al. Automated, high-throughput derivation, characterization and differentiation of induced pluripotent stem cells. Nature methods. 12 (9), 885-892 (2015).

10. Busskamp, V. et al. Rapid neurogenesis through transcriptional activation in human stem cells. Molecular systems biology. 10, 760 (2014).

11. Zhang, Y. et al. Rapid single-step induction of functional neurons from human pluripotent stem cells. Neuron. 78 (5), 785-798 (2013).

12. Reinhardt, P. et al. Derivation and expansion using only small molecules of human neural progenitors for neurodegenerative disease modeling. PloS one. 8 (3), e59252 (2013).

13. Kriks, S. et al. Dopamine neurons derived from human ES cells efficiently engraft in animal models of Parkinson's disease. Nature. 480 (7378), 547-551 (2011).

14. Koch, J.C. et al. Alpha-Synuclein affects neurite morphology, autophagy, vesicle transport and axonal degeneration in CNS neurons. Cell death \& disease. 6 , e1811 (2015).

15. Korecka, J.A. et al. Neurite Collapse and Altered ER $\mathrm{Ca} 2+$ Control in Human Parkinson Disease Patient iPSCDerived Neurons with LRRK2 G2019S Mutation. Stem cell reports. 12 (1), 29-41 (2019).

16. Mehta, S.R. et al. Human Huntington's Disease iPSC-Derived Cortical Neurons Display Altered Transcriptomics, Morphology, and Maturation. Cell reports. 25 (4), 1081-1096.e6 (2018).

17. Ye, J., Coulouris, G., Zaretskaya, I., Cutcutache, I., Rozen, S., Madden, T.L. Primer-BLAST: a tool to design target-specific primers for polymerase chain reaction. BMC bioinformatics. 13, 134 (2012). 
18. Spandidos, A., Wang, X., Wang, H., Seed, B. PrimerBank: a resource of human and mouse PCR primer pairs for gene expression detection and quantification. Nucleic acids research. 38 (Database issue), D792-9 (2010). 